\title{
Del análisis de las prácticas territoriales al diseño de políticas públicas. Recorridos y escenarios de la Ley de Niñez provincial en La Plata
}

From de analysis of territorial practices to the design of public policies.

New paths and sceneries of the childhood provincial law in La Plata

Anabel Urdaniz anabel_urdaniz@hotmail.com

http://orcid.org/0000-0002-1630-5074

Facultad de Periodismo y Comunicación Social; Instituto de Investigaciones en Comunicación;

Universidad Nacional de La Plata/ Comisión de Investigaciones Científicas (Argentina)

\section{Resumen}

El presente trabajo es un breve ensayo que actualiza el libro de mi autoría Nuevos recorridos y escenarios en el marco de la Ley de Niñez Provincial 13298. Una mirada comunicacional en torno a las prácticas territoriales de los Servicios Locales de Niñez de Altos de San Lorenzo y Villa Elvira. Existen varias situaciones políticas en relación al territorio que configuran la 
práctica académica. En 2016, luego de dos años de recibirme con la tesis de grado con el mismo nombre, la gestión de Julio Garro cerró los dos Servicios locales de Promoción y Protección de los Derechos de la Niñez, que eran objeto de estudio de la investigación. Devino entonces como necesidad inminente la publicación en formato libro del trabajo.

Después de dos años, la política de la alianza Cambiemos a nivel nacional, provincial y municipal ha presentado evidencias de la corrosión lenta del paradigma de promoción y protección por otros paradigmas meritocráticos como el del Capital Mental. Este artículo pretende indagar la complejidad de las prácticas territoriales y su relación con el diseño de políticas públicas para repensar otras variables que las conforman, que no sólo se evidencian en el ajuste o el recorte presupuestario y que también están siendo corroídas simbólica y físicamente.

Palabras clave: Políticas públicas; niñez; prácticas; territorio; representación.

\section{Abstract}

This work is a brief essay that updates the book of my authorship called autoría Nuevos recorridos y escenarios en el marco de la Ley de Niñez Provincial 13298. Una mirada comunicacional en torno a las prácticas territoriales de los Servicios Locales de Niñez de Altos de San Lorenzo y Villa Elvira. There are several political situations related to the territory that configure the academic practice. In 2016, two years after my graduation with the degree thesis which was so called after my book, Julio Garro' s government, closed both Local Services for the Promotion and Protection of Children's Rights, which were the source of subject investigation. Consequently, there came the urgent publication of the work in book format.

After two years, the political ideas of the current Cambiemos alliance at all the national, provincial and local levels have shown remarkable evidence of slow corrosion of the promotion and protection paradigms in exchange for other meritocratic paradigms such as Mental Capital. This article has as an objective to investigate the complexity of the territorial practices and their relationship with the design of public policies, in order to rethink other variables that comprise them. These variables are not only evident due to a shortening in the budget but also to a symbolic and physical corrosion.

Keywords: Public policies; childhood; practices; territory; representation. 
El nuevo paradigma de promoción y protección de los derechos de la niñez lo inaugura en 1989 la Convención Internacional sobre los derechos de la niñez, ratificada por los 196 estados que conforman la Asamblea de las Naciones Unidas, con excepción de Estados Unidos. El Tratado es vinculante respecto a las legislaciones de los países. Se trata del primer documento que establece que los niños y las niñas son sujetos de derechos y que en cualquier conflictoambiental, político, económico, internacional- debe primar el interés superior del niño o la niña. Argentina adhiere a la Convención y la introduce a la Constitución con la Reforma de 1994. Desde ese momento, las organizaciones de niñez y diferentes actores claves comienzan a organizarse para luchar por una Ley Nacional acorde a la Convención.

En 2005 se sanciona la Ley Nacional de Protección Integral de los derechos de los niños, niñas y adolescentes Nro. 26061, normativa que era sumamente necesaria ya que en algunas provincias ya se habían promulgado leyes acordes con la Convención como la Ley 6354 en 1995 en Mendoza; la 4347 en Chubut en 1997; la 114 en CABA en 1998 o la 2302 en Neuquén, en 1999. En lo que respecta a la provincia de Buenos Aires, en 2006 se decreta la Ley provincial de Promoción y Protección de los Derechos de la Niñez Nro. 13298. Si bien, no fue una de las primeras, cabe mencionar que tiene algunas particularidades de avanzada respecto a las otras: establecimiento de tiempos en los procesos, creación de organismos coparticipativos, ponderación de lo territorial como fundamento clave de toda acción, diferenciación de sistemas, entre otras.

\section{Coordenadas actuales sobre la situación de la Niñez}

Según estadísticas de 2018 del Barómetro de la Infancia de la UCA (1), en la Argentina 8.255.000 niños, niñas y adolescentes se encuentran en situación de pobreza. Entre 2016 y 2017 la pobreza de la franja etaria creció un 2,1\% pasando de $60,4 \%$ a $62,5 \%$. Entre las principales privaciones, el informe destaca la alimentación, las condiciones de hábitat, el acceso a la educación y la salud. Dicho panorama se ve afectado profundamente por el convenio con el Fondo Monetario Internacional, lo que ya significó recortes concretos en las áreas que deben financiar el sistema nacional como la Secretaría Nacional de Niñez, Adescencia y Familia (SENNAF). El 7 de septiembre la Jefatura de Gabinete de Ministros, a cargo de Marcos Peña, expidió la decisión administrativa 1605/2018 (2) que indica en forma literal el recorte presupuestario de áreas sustanciales para sostener el Sistema. Las organizaciones sociales (3) estiman que se trata de un recorte que oscila los $\$ 124.000 .000$, de los cuales 115 millones son recortes a programas de Primera Infancia de 0 a 4 años. 
En lo que respecta a la provincia de Buenos Aires, la Secretaría de Niñez y Adolescencia desde 2016 perdió el rango para convertirse en Organismo de Niñez y Adolescencia bajo la órbita de Desarrollo Social. Según datos de 2018 de la Subsecretaría de Hacienda (4), del total del presupuesto sólo se le destina a Desarrollo Social el $2.57 \%$, lo que implican $\$ 16.206 .566 .025$, mientras que al Organismo le destinan $\% 14,58$ de ese total, lo que da \$2.363.196.552. De ese total un $18 \%$ se destina a obras, un $40 \%$ al Sistema de Responsabilidad Penal Juvenil y un $41 \%$ al Sistema de Promoción y Protección de Derechos, quedando un total de dinero para atención directa de $\$ 984.474 .000$. El Organismo sólo recibe un $0.37 \%$ del total del presupuesto provincial.

En lo que atañe a los municipios, al crearse la Ley 13298 se estableció que el financiamiento de este nuevo sistema se realizaría con un Fondo de Fortalecimiento provincial de Programas Sociales establecido por la Ley 13163 y su decreto reglamentario 609/04. Dicho dinero que representa la recaudación de los inmobiliarios rurales y juegos de azar, entre otros, nunca llega a las Secretarías o Direcciones de Niñez, sino que se emplea en otras necesidades que establecen las carteras municipales de Desarrollo Social. Desde que el paradigma de promoción y protección se inaugura en nuestro país, las organizaciones sociales e instituciones cercanas al tema, solicitan una Ley de Financiamiento del sistema de Niñez con inversión en políticas públicas e intangibilidad de los Fondos. Si bien, no existen relevamientos oficiales, el último Informe de la Comisión provincial por la Memoria en 2018, revela que el $45 \%$ de los municipios no tienen programas propios de niñez, el 36\% sólo tiene un programa propio y el $19 \%$ tiene dos o más programas. El $50 \%$ de los municipios tienen los Servicios Locales de Niñez, organismo de atención directa, centralizado, con escasos recursos y sin computadoras o internet necesario para cargar los datos de las situaciones.

Los y las funcionarias no ven a la niñez como una temática estratégica, lo que se observa año a año en la desfinanciación del Sistema de Promoción y Protección. Fue una decisión política comenzar con estos datos que subyacen las prácticas que se pretenden analizar y las condicionan cotidianamente.

\section{Anteojeras teóricas}

La mirada comunicacional de la tesis adscribe a los Estudios Culturales Latinoamericanos (Martín-Barbero, 1987; García Canclini, 1996; Schmucler, 1984; González, 1992), lejos de pensar la comunicación centrada en los medios masivos o en productos comunicacionales, se la concibe como construcción social de sentido habilitante de ciertos discursos que constituyen 
prácticas sociales estructurando instituciones y dinámicas sociales. "Se trata de una acción colectiva, organizada o no, en la que intervienen varios actores poniendo en juego sus saberes, intereses y poderes, generando formas de interacción que provocan consecuencias sociales, económicas, culturales y políticas" (Uranga, 2012: 15) Ergo, hablamos de leer las prácticas territoriales como "prácticas de enunciación", que generan recorridos y tramas que sedimentan marcos de referencia o "claves de interpretación" (Uranga, 2012). Las prácticas sociales son formaciones sociales históricas que tienen un insumo, o lo que podríamos llamar "texto" pero que dicha "marca de racionalidad" (Massoni, 2017) no sería nada, es decir, no podría explicarse sin el contexto, "por ello las prácticas son diversas, libres y azarosas como el ser humano" (Prieto Castillo, 1990: 105).

Desde esta perspectiva, la cultura no es lo culto sino dimensión significante de lo social que tiene materialidad en distintos soportes sociales. La comunicación de esta forma es momento enactuante, hace emerger realidades y escenarios posibles. Así, la comunicación es transferible a la vida cotidiana. Por lo que el gran esqueleto de la investigación lo constituyen los actores sociales situados y los territorios que los estructuran y que, a su vez, se ve modificado por la interacción con sus prácticas. Los actores sociales son unidades reales de acción, al ser locales tienen dominios legítimos que han sabido construir en relación a la gestión de recursos, a la construcción sectorizada de demandas y a la articulación barrial. Este trabajo no desconoce la economía general de las prácticas (Bourdieu, 1991), sino que a medida que avanza la investigación intenta recuperar cuáles son esas disposiciones que se regularizan en las prácticas.

El territorio está pensado como lugar habitado por el pasado, el presente y el futuro, lugar de temporalidades que se reconfiguran en las prácticas, por lo que se lee el territorio como lo físico o material y como simbólico e imaginario. "Cada barrio es un texto a leer, escritos que hablan de construcciones simbólicas de quienes los habitan, de cómo se construye sentido en la vida cotidiana, desde cimentar significados hasta resolver problemas de la práctica" (Carballeda, 2008: 73). Para hacer asequible el territorio, que reviste una materialidad concreta, se trabajó en base a mapas con nomenclaturas oficiales pero configurándolos con las representaciones que los actores sociales hacían de los lugares en los que vivían. Para ello, se mapearon escenarios, trayectorias y fronteras físicas y simbólicas.

Es en la intersección del entramado conceptual desplegado, donde aparece la noción de identidad, que no se piensa como una esencia ni como algo material que pueda ser obtenido 0 perdido. Se trata de elementos de la cultura internalizados y legitimados por grupalidades de pertenencias que se ordenan en base a alteridades $u$ otredades. "Identidad, en términos sociológicos, es el proceso por el cual los actores sociales construyen el sentido de su acción 
atendiendo a un atributo cultural (o un conjunto articulado de atributos culturales) al que se da prioridad sobre otras fuentes posibles del sentido de la acción" (Castells, 1999: 4) En este sentido se trabajaron los "efectos de lugar" (Bourdieu, 1991), la descentralización de las políticas públicas como correlato nacional, provincial y municipal y la corporización del territorio.

\section{Cartografías físicas y simbólicas: Altos de San Lorenzo y Villa Elvira}

La elección de los territorios estuvo relacionada, en primer lugar, con la cantidad de situaciones de niños y niñas con derechos vulnerados. Para establecer estos datos, se realizaron algunas entrevistas al ex Director de Niñez del Municipio platense, Carlos Davaleoni, quien aseguró que según el Registro Estadístico Único de Niñez y Adolescencia (REUNA), las dos delegaciones que más casos recibían eran Altos de San Lorenzo y Villa Elvira.

Altos de San Lorenzo se encuentra al sudeste del casco platense, comenzó a poblarse entre 1940 y 1950. En su mayoría, los habitantes eran los trabajadores del Ferrocarril, puesto que en la década de 1970 se encontraba en 17 y 72 la Estación Central de Ferrocarril Provincial. Según datos del censo 2001 (5), vivían allí 30.912 personas, de los cuales casi la mitad son niños y niñas, $31 \%$ son varones y $8.9 \%$ son mujeres de 0 a 14 años. Más de 8 mil habitantes se encontraban inactivos o desempleados y 6759 personas tenían necesidades básicas insatisfechas.

Al momento del análisis, el Servicio Local de Niñez se encontraba desde 2009 en el Centro de Integración Barrial en 18 y 21(6), donde se llevaban a cabo variedad de actividades de diferentes instituciones que convivían en ese espacio. El equipo de profesionales está compuesto por una psicóloga, una trabajadora social, una abogada y un operador de calle.

"Trabajan en una pieza de tres por cuatro, en la que hay un gran escritorio en el centro, repleto de papeles. No tienen teléfono, sino que lo comparten con todo el Centro de Integración Barrial, tienen una computadora de escritorio y una computadora que consiguieron hace una semana. No hay espacios para guardar legajos, no hay juguetes, las paredes son grises" (Nota de campo, septiembre de 2012).

Villa Elvira es una delegación más antigua, fue parcelada y habitada 1908 pero se fundó en 1940. Se extiende desde la avenida 72 hasta Magdalena entre la avenida 13 y el límite con la ruta provincial Nro. 11. La población total es de 59476 habitantes, de los cuales el $51 \%$ son varones y mujeres de 0 a 14 años. Más de 9 mil habitantes tienen en Villa Elvira necesidades básicas insatisfechas, es decir que más del 17\% vive hacinado, con hogares precarios, con 
paupérrimas condiciones de higiene y sin capacidad de subsistencia, según algunos de los parámetros del INDEC. En este caso, el Servicio Local de Niñez también fue creado en 2009 y se encuentra en 7 entre 76 y 77 en un local partidario, cuya inscripción ni bien ingresas indica "Acá hay equipo Pacha". Trabajan allí una psicóloga, una trabajadora social, un abogado y un operador de calle, que es despedido en el transcurso de la investigación (7).

En el caso de Altos de San Lorenzo se trabajó con las instituciones: Programa provincial Barrio Adentro, Centro de Prevención de Adicciones, Escuela Primaria Nro. 40, Centros de Salud Municipales Nro. 41 y Nro. 8, Programa de Juegotecas barriales, Unión de Cartoneros y Mesa local de Niñez. En Villa Elvira se trabajó con el Centro de Salud Nro. 43, la Escuela Primaria 125 y la 23, Casa Joven y la Mesa barrial de Niñez.

\section{Matrices de análisis}

Con el objetivo último de analizar las prácticas territoriales de los Servicios locales de Niñez, se crearon tres matrices de análisis que atravesaron la investigación. Dichas matrices funcionaron como ordenadores o guías de búsqueda así como configuradoras del posterior análisis comparativo constante entre las dos delegaciones. La primera matriz tiene como objetivo configurar las dinámicas de ambos barrios, se llama "identidad barrial" y está dividida en las dimensiones: fronteras físicas, fronteras simbólicas, conflictos sociales y presencia institucional. La segunda matriz pone el foco en el andamiaje institucional que existe en cada uno de los territorios, se llama "Representación de las prácticas institucionales de niñez en el marco de la nueva ley" y presenta tres dimensiones de análisis, que a su vez se subdividen en variables. Presencia institucional que se divide en la incidencia territorial de la institución y la participación comunitaria, por lo que se subdivide en las variables: origen de la institución, referencia de la institución en el barrio, conocimiento del barrio por parte de la institución, población abordada, permanencia institucional, nivel de legitimidad como el poder en la resolución de los casos y el dominio de las situaciones y participación de los vecinos en la institución. Otra de las dimensiones organiza la "Metodología de abordaje de la institución" y está dividida en las variables o sub-dimensiones: recursos, acción ante la urgencia o emergencia, mirada tutelar o de protección integral, acción en la prevención, protección o restitución de derechos, saberes y destrezas. La última dimensión de la matriz indaga las "relaciones institucionales", las sub dimensiones son: espacios compartidos, niveles de poder y jerarquías entre instituciones, obstáculos para los encuentros y noción de corresponsabilidad y red en sus discursos y en su accionar. 
Representación de las prácticas institucionales de ninèz en el marco de la nueva ley.

\begin{tabular}{|c|c|c|c|c|c|c|}
\hline \multicolumn{6}{|c|}{ Presentación Institucional- Incidencia territorial y participación comunitaria } & \multirow{2}{*}{$\begin{array}{l}\text { Participación de los } \\
\text { vecinos en la inst. }\end{array}$} \\
\hline $\begin{array}{l}\text { Sub- } \\
\text { dimensiones }\end{array}$ & $\begin{array}{l}\text { Orizen/nacim } \\
\text { de la } \\
\text { institución }\end{array}$ & $\begin{array}{l}\text { Referencia de la inst en el } \\
\text { barrio/conos del barrio por } \\
\text { parte de la inst. }\end{array}$ & $\begin{array}{l}\text { Población } \\
\text { abordada }\end{array}$ & Permanencia institucional & $\begin{array}{l}\text { Nivel de legitimidad (poder en la } \\
\text { resolución de casos y dominio de } \\
\text { situaciones). } \\
\text { Sivel de incidencia }\end{array}$ & \\
\hline $\begin{array}{l}\text { Barrios } \\
\text { Adentro }\end{array}$ & $\begin{array}{l}\text {-Se crea por } \\
\text { descentralizaci } \\
\text { on del SAF, } \\
\text { pura acompaihr } \\
\text { a jovenes con } \\
\text { responsabilidad } \\
\text { penal. } \\
\text {-Asumen que } \\
\text { son un } \\
\text { programa } \\
\text { etatal. } \\
\text { Representantes } \\
\text { del estado. }\end{array}$ & $\begin{array}{l}\text {-Existen diagnosticos creados } \\
\text { por la institucion } \\
\text { - Comienzan a trabajar en el } \\
\text { barrio a partir de un recoerido } \\
\text { por tribunales. } \\
\text { - La poblacion lo referencia } \\
\text { como una organizacion con la } \\
\text { que se puede contar, que } \\
\text { resuelve. } \\
\text { - Se suman con el tiempo } \\
\text { miembros que vienen de } \\
\text { distintas organizaciones del } \\
\text { barrio pero que no viven en el. } \\
\text {-Utilización de espacios en los }\end{array}$ & $\begin{array}{l}\text { Aproximadam } \\
\text { ente } 40 \text { chicos } \\
\text { son abordados } \\
\text { por el } \\
\text { programa } \\
\text { - Gran } \\
\text { conocimiento } \\
\text { de su } \\
\text { existencia en } \\
\text { el barrio. }\end{array}$ & $\begin{array}{l}\text { - Existen profesionales que estin } \\
\text { en el barrio desde el } 2007 \text {, pero } \\
\text { Barrios Adentro comienza en el } \\
2008 \text {. Su trabajo en el bartio ha } \\
\text { sido continuo. }\end{array}$ & 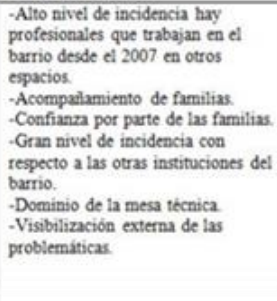 & $\begin{array}{l}\text {-Ingresan al barrio } \\
\text { mediante tres referentes } \\
\text { que poseen comedores. } \\
\text { - Fuente participacion de } \\
\text { vecinos. } \\
\text {-Apropiacion de nuevos } \\
\text { espacios elegidos por los } \\
\text { nitios cerca de las casa de } \\
\text { sus familias. } \\
\text {-Si surgen nuevas } \\
\text { problemiticas buscan } \\
\text { trabajar en conjunto con } \\
\text { familias o instituciones. } \\
\text { Son flexibles. }\end{array}$ \\
\hline
\end{tabular}

Figura 1. Matriz de análisis propia sobre la "Representación de las prácticas institucionales de niñez".

Finalmente, la última matriz se titula "Representaciones de las prácticas territoriales de los Servicios Locales de Niñez", para analizarla se subdividió en las representaciones respecto a lo que se considera derecho vulnerado y cómo esa configuración guía el accionar, a través de las variables: urgencia y emergencia, recorte de la demanda y acción desde la prevención, promoción o restitución. La segunda dimensión de esta matriz puso el foco en la metodología de abordaje, sus variables son: recursos, estrategias de abordaje, trabajo territorial, participación de los niños y niñas, acciones, principales obstáculos. La última dimensión trabajo las relaciones de los Servicios locales con las otras instituciones abordadas, las variables en este caso se organizaron de la siguiente forma: representación del servicio local en el territorio ("en el barrio se comenta"), espacios de encuentro con otras instituciones, nivel de incidencia del Servicio Local en el barrio respecto a la resolución de casos, noción de red, obstáculos para fomentar la corresponsabilidad.

Es importante señalar que dichas matrices se configuraron a partir de las representaciones que surgían de los discursos de las trece instituciones relevadas, de los Servicios Locales de Niñez de cada delegación y también de los vecinos y las vecinas del barrio. Se trabajó con entrevistas en profundidad, entrevistas semi formales, observaciones no participantes, observaciones participantes, cuaderno de campo, encuestas y diferentes tipos de mapeos. La última técnica fue de gran utilidad y se ampliará en un apartado más avanzado el análisis.

Las anteriores matrices constituyeron el esqueleto de este arduo trabajo de tesis, a continuación se desarrollarán algunas de las aristas que más sobresalieron de cada una. En el caso de querer leer cada una de las dimensiones de análisis en profundidad, se adjunta el libro y los anexos en el siguiente enlace (8).

Question, Vol. 1, N. ${ }^{\circ}$ 61, enero-marzo 2019. ISSN 1669-6581

Instituto de Investigaciones en Comunicación | Facultad de Periodismo y Comunicación Social | Universidad Nacional de La Plata La 


\begin{tabular}{|c|c|c|c|c|c|}
\hline \multirow{2}{*}{$\begin{array}{l}\text { Actores } \\
\text { Sub- } \\
\text { dimensiones }\end{array}$} & \multicolumn{4}{|c|}{ Metodologia de abordaje } & \multirow{2}{*}{\begin{tabular}{|l|} 
Saberes y destrezas \\
\end{tabular}} \\
\hline & Recursos & Urgencia/emergencia & Mirada tutelar o de protección integral & Acción en la prex. Prom o restituc. & \\
\hline $\begin{array}{l}\text { Barrios } \\
\text { Adentro }\end{array}$ & $\begin{array}{l}\text {-Cantidad de } \\
\text { instituciones en el } \\
\text { barrio. } \\
\text {-Nivel de redes. } \\
\text { - profesionales } \\
\text { interdisciplinarios. } \\
\text { Una oficina } \\
\text { centralizada y dos } \\
\text { espacios en el barrio } \\
\text { de reunión. } \\
\text {-Caja chica de } \\
\text { provincia destinada al } \\
\text { programa. } \\
\text {-Realizan balances } \\
\text { todos los años. }\end{array}$ & $\begin{array}{l}\text {-No esperar a la } \\
\text { emergencia, "salir a la } \\
\text { calle". } \\
\text {-Generar propuestas que } \\
\text { den confianza y habiliten } \\
\text { espacios. }\end{array}$ & $\begin{array}{l}\text {-El programa se crea según los } \\
\text { lineamientos de la nueva ley de niñez "La } \\
\text { ley tiene un espiritu de desandar } \\
\text { practicas" } \\
\text {-Mirada de promoción y protección } \\
\text { integral. Haciendo hincapié en la } \\
\text { promoción. } \\
\text {-Mirada territorial y de fortalecimiento de } \\
\text { lo vincular. } \\
\text {-"Los vínculos flojos hacen que haya cada } \\
\text { vez más chicos institucionalizados". }\end{array}$ & $\begin{array}{l}\text {-Trabaja en los tres momentos pero pone } \\
\text { énfasis en la promoción: generar } \\
\text { circuitos alternativos a partir de } \\
\text { necesidades del barrio. } \\
\text { "Dispositivos flexibles". }\end{array}$ & $\begin{array}{l}\text {-Talleres, murales, espacios } \\
\text { recreativos que perduran en el } \\
\text { tiempo y generan identidad. } \\
\text {-Fortalecimiento de redes } \\
\text { como la mesa técnica. } \\
\text {-Inclusión de actores externos ( } \\
\text { ej: universidad) al barrio. }\end{array}$ \\
\hline
\end{tabular}

Figura 2. Matriz de análisis propia sobre la "Metodología de Abordaje" de las instituciones de niñez.

\section{Identidad barrial, mapeando problemáticas}

Para entender las prácticas territoriales de los Servicios locales devino fundamental comprender las dinámicas del "barrio" (como llaman todos los actores entrevistados a la delegación). Pero no sólo comprender las coordenadas materiales: las líneas de colectivos, los lugares con más presencia institucional, las fronteras físicas como baldíos, basurales y asentamientos, sino además realizar un análisis respecto a la representación de ambas delegaciones por parte de los que la transitan y la hacen cuerpo. De ambas delegaciones se diseñaron mapas cartográficos con la nomenclatura de dichas fronteras (9).

"Faltan seguimiento de las niñas embarazadas, algunas están por tener y aun no tienen ningún control, este fenómeno se repite y aumenta" (Representante del Centro de Salud Nro. 8).

Entre las principales problemáticas en Altos de San Lorenzo se evidencia un fuerte conflicto habitacional, existen muchas peleas intra vecinales por los asentamientos que irrumpen el trazado de calles de 81 a 90 y de 14 a 19. La estigmatización hacia las personas que viven en los asentamientos se profundiza por la condición de inmigrantes, por lo que se han podido delimitar a estos asentamientos como barreras simbólicas estigmatizantes. La situación habitacional se agrava en 2013 con la inundación catastrófica acontecida el 13 de abril. Al tratarse de una zona semi rural, existen muchos terrenos desnivelados y no existe planificación urbana que implique obras de desagüe, cloacas, agua corriente o recolección de basura. Otra de las preocupaciones es la cantidad de animales que deambulan por las calles y las 
enfermedades que contraen, por lo que se han realizado varias campañas de zoonosis municipales.

Además, irrumpen como fronteras físicas una cantidad de terrenos desocupados, entre ellos se encuentra la cantera que se extiende de 81 a 90 y de 15 a 16 y los baldíos que van desde 76 a 80 y de 23 a 25. Dichas interrupciones no sólo configuran los recorridos del escaso transporte público sino que generan una división respecto a las instituciones que están más cerca de "la avenida 72" de las que están del otro lado de este gran baldío más cerca de "Puente de Fierro". Se llama así a la última zona habitada de la delegación en las calles 90 y 29 en donde se ubica un enorme puente que quedó de la época en que por allí pasaba el ferrocarril; esa zona es una de las más precarias de la delegación.

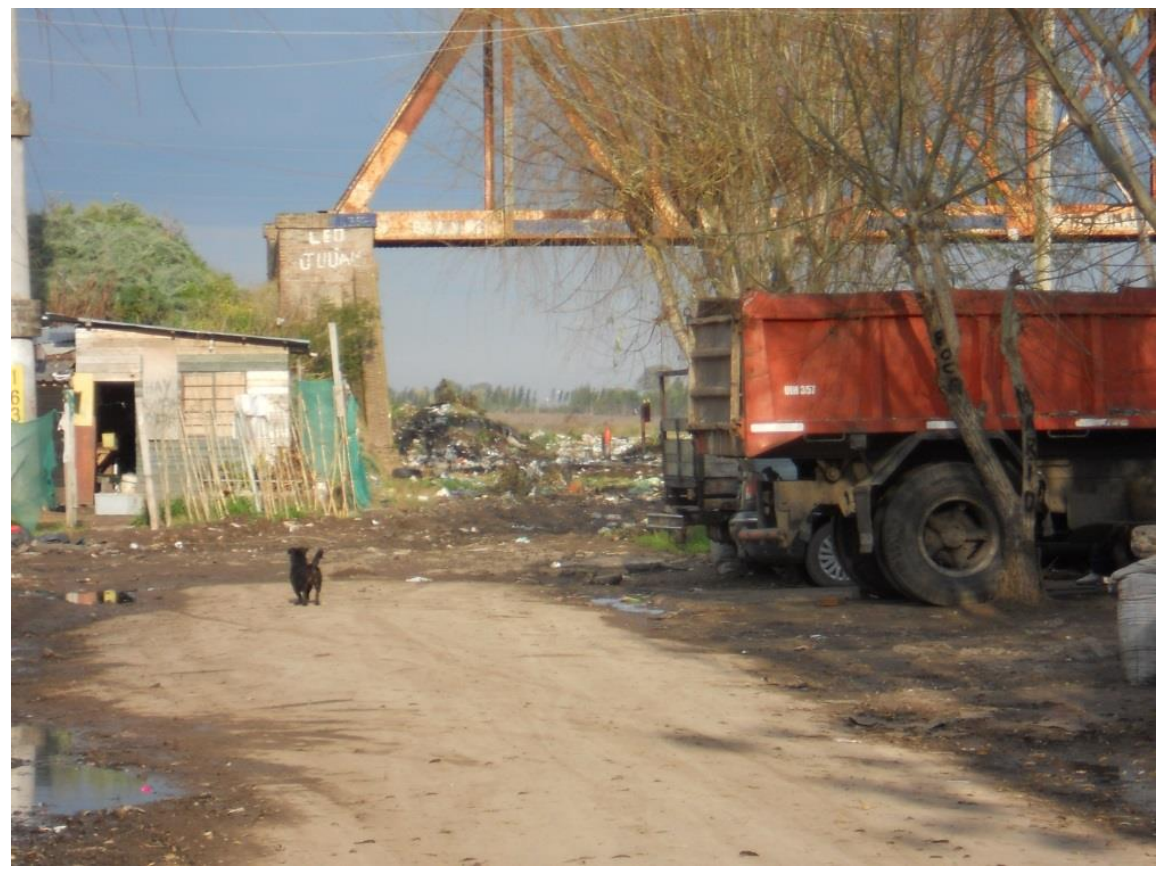

Figura 3. Puente de Fierro, 2013. Producción propia.

A medida que uno pasa el descampado, las calles dejan de ser asfaltadas, mientras un montón de casas precarias se ubican a orillas del arroyo Maldonado, sumado a que debajo del puente hay un gran basural a cielo abierto donde los vecinos depositan sus desechos debido a que ahí no llega el recolector. Existe un mapa mental de abordaje, en el que los niños y niñas que están "allá" son abordados por otras instituciones, por ejemplo por el Centro de Salud Nro. 41 o por la escuela Nro. 22 o Nro. 60, mientras que si la situación se da entre las calles 72 y 85 el abordaje es de "acá", que es el lugar donde se encuentra el Servicio local de Niñez y donde 
coordina con varias instituciones de las entrevistadas que están de este otro lado (más cerca de la 72), como el Centro de Salud 8, la Escuela 40 o la Unión de Cartoneros.

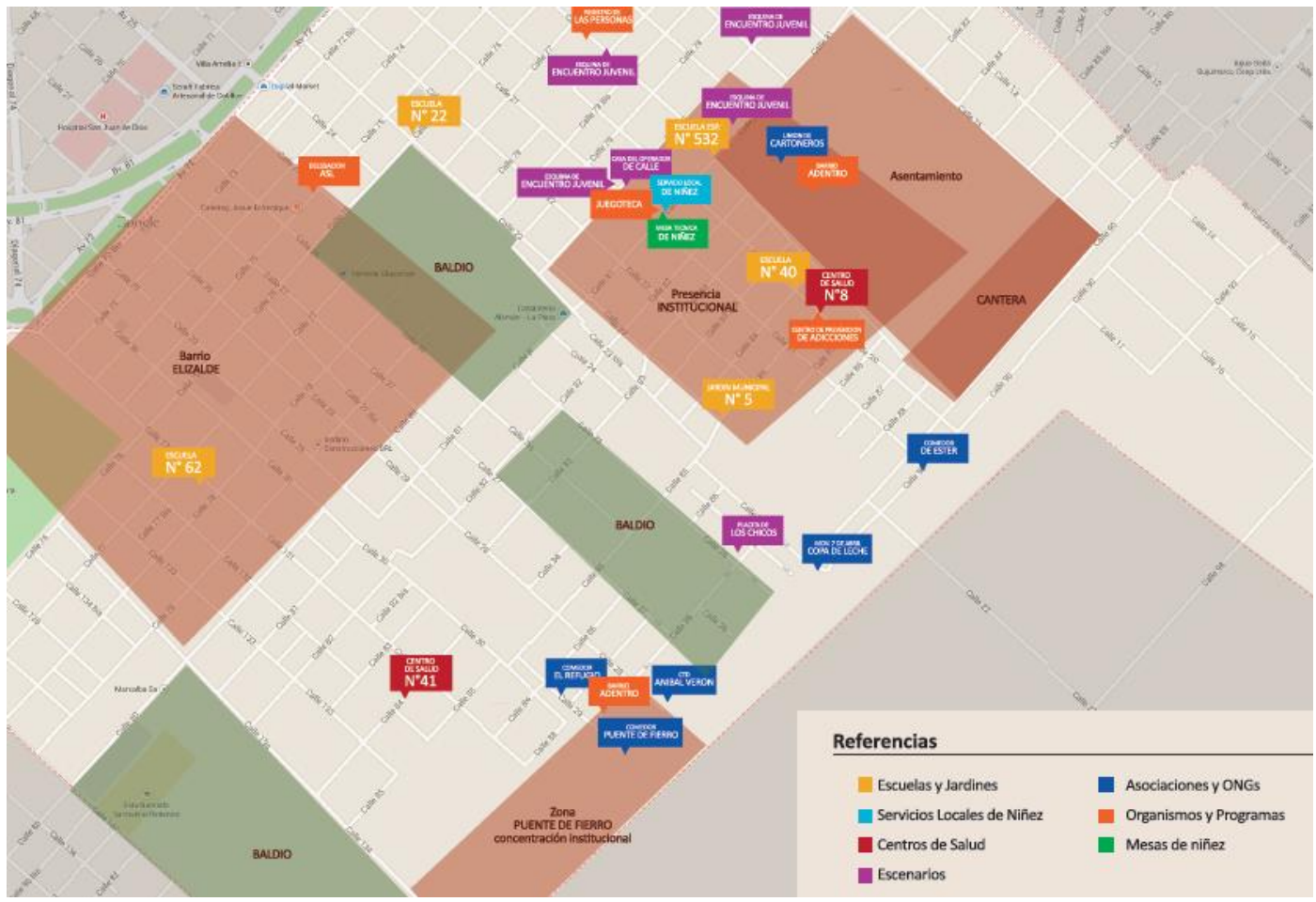

Figura 4. Mapeo de producción propia de las instituciones y escenarios de Altos de San Lorenzo (Recuperado de https://goo.gl/JNJ37c)

Según las instituciones entrevistadas se evidencian muchos casos de violencia intrafamiliar, entre lo que resalta el maltrato físico y abuso sexual. Se observó además un elevado número de niños y niñas que llegan a las instituciones con algún tipo de discapacidad. En este sentido, desde los Centros de Salud afirman que en muchas oportunidades los niños o niñas llegan con alguna urgencia como una quemadura y ahí es donde se descubre algún tipo de retraso madurativo. Esto evidencia la falta de atención primaria de la salud y junto a este tema, la falta de programas de educación sexual integral. Desde el Centro de Prevención de Adicciones nos comentan que existe consumo problemático de adolescentes y que en su mayoría no se acercan al lugar por lo que cuesta hacer seguimiento. Al respecto los vecinos indican que "en el barrio están los circuitos" y que para los chicos es muy difícil salir de ahí. Además, en relación al tema, indican cuáles son las "esquinas problemáticas" donde los jóvenes se juntan a pasar el rato y consumir. 
"Uh... los pibes se juntan a tomar en varias esquinas: 17 y 80,80 y 21,81 y 19 , y 78 y 19 , es medio peligroso" (Vecina de Altos de San Lorenzo).

El correlato de esta situación es la falta de espacios recreativos para los adolescentes y jóvenes. Mientras se realizaba la investigación (2012-2014) en la delegación no había escuela secundaria con edificio propio, sino que debían compartir aulas con las primarias.

\section{Villa Elvira}

La delegación se encuentra dividida en barrios que han sabido generar marcas identitarias, lo que se observa en la construcción de los recorridos que se encuentran diferenciados por la localización de los barrios, entre los que figuran los más nombrados como Jardín, Monasterio, Villa Alba, Aeropuerto y El Palihue.

El barrio al que pertenecen también determina la escuela a la que asisten, una maestra afirma que la mayoría de los que viven en Aeropuerto no van a la escuela 23 ubicada en 7 y 82 , mientras que desde Casa Joven ubicado en 97 entre 6 y 7 tienen como principal actores a los jóvenes que vienen desde ese barrio.

Como en Altos de San Lorenzo, también se observan interrupciones en la urbanización por grandes lotes de terreno que se usan para basural. Uno de los lugares más peligrosos de la delegación son las canteras, que se ubican en 1 y 631 y otra en 122 y 615 . Se trata de lugares donde se extrae tierra, especie de piletas enormes, donde se tiran residuos pero a su vez cuando llueve se llenan de agua, por lo que no sólo son foco de contaminación, sino también lugar muy peligroso para los niños y las niñas que juegan allí habitualmente. Entre 2010 y 2012 murieron ahogados dos niños en esas canteras, la problemática esta naturalizada por parte de los vecinos. 


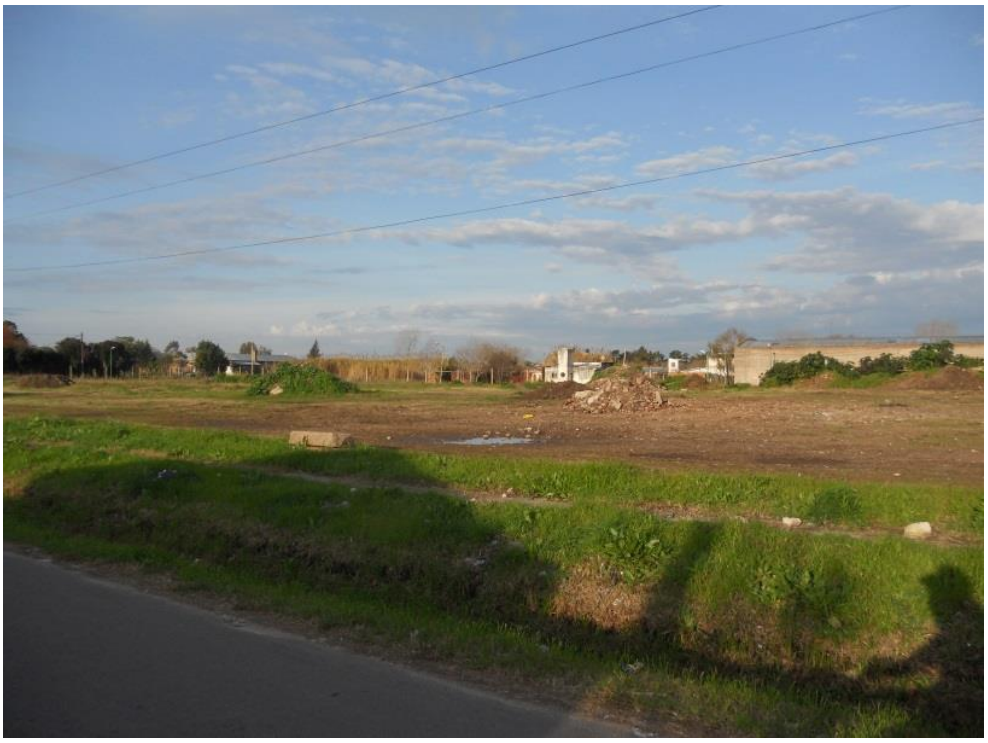

Figura 5. Fotografía de producción propia, descampado en 1 y 80.

Entre los espacios que intervienen en las trayectorias se encuentra la Unidad Carcelaria Nro. 9, el Aeropuerto, el paso del arroyo Maldonado y varios vacíos urbanos, uno de los más grandes: el que va de 116 hasta avenida 7 y desde 84 hasta la 90 . Retomando el concepto de "efectos de lugar" de Pierre Bourdieu, así como la corporizarían de la pobreza de Julia Arechaga (2011), a medida que uno pasa la 90 y camina de 7 hacia 1 se empieza a observar la falta de numeración, la precariedad de las casa aumenta así como su tamaño, se ve constantemente humo de fogatas de basura o bien brea quemada y cantidad de gente sentada en sus casas como esperando. Alli no se observa presencia del Estado, no hay carteles políticos, ya que ni siquiera hay postes de luz para colgarlos, hay pequeños almacenes en las entradas de las casas, que en su mayoría son de chapas y sólo algunas paredes de material.

"La calle 630 hace las veces de límite, de la 630 para allá ya es Arana y está complicado" (vecino de Villa Elvira).

Esta "peligrosidad" constituye una barrera simbólica que actúa directamente sobre los recorridos. Por ejemplo, hasta ahí llegan las maestras. Si las familias viven después de la 630 los citan en la escuela o los llaman por teléfonos. Tampoco entran ambulancias ni otros referentes de organizaciones. 


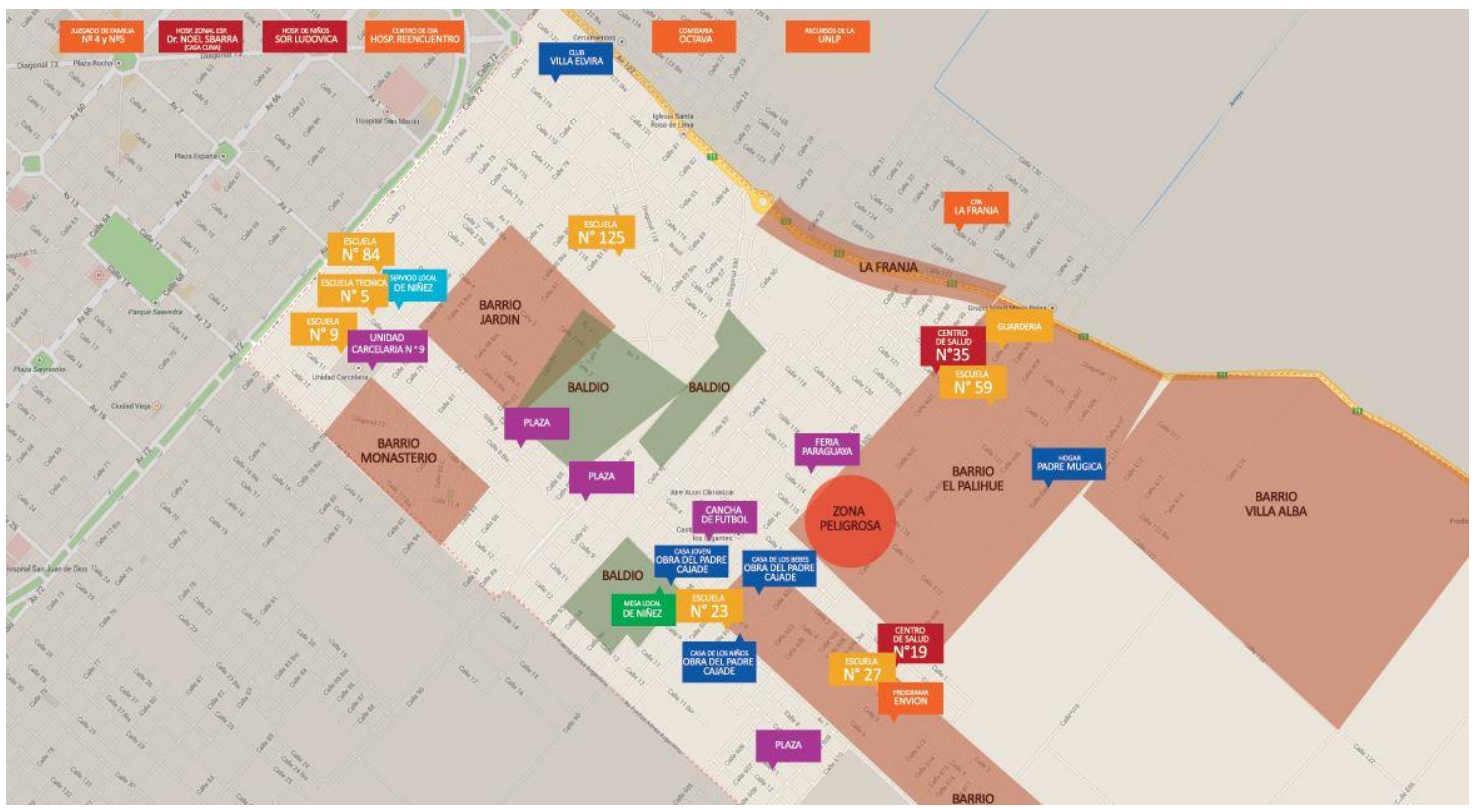

Figura 6. Mapeo de producción propia de las instituciones y escenario de Villa Elvira

(Recuperado de https://bit.ly/2CyMkYm)

Como problemáticas sociales se pueden observar la violencia intrafamiliar- lo que se agrava por las situaciones estructurales- y el consumo problemático de drogas. Entre las frases que nos dicen los vecinos o familiares, resuena "los jóvenes deambulan" lo que consciente o inconscientemente está hablando de la no pertenencia, la no estabilidad, la no intervención del estado en pos de generar espacios en donde se puedan identificar. En este sentido, cabe resaltar que no observamos estigmatización por parte de los vecinos en relación a "estos jóvenes" sino que tienden a problematizar la situación y a culpabilizar en general al padre y a la madre: "y vos, que querés que yo haga", le decía una señora al operador de niñez durante una citación; "si yo soy la abuela, acá están los circuitos. La única solución es que salga del barrio". Existen también muchas situaciones de niños o niñas con retraso madurativo o diferentes discapacidades que las trabajadoras atribuyen al hambre 0 a las condiciones básicas insatisfechas.

Otra de las problemáticas que se observa es la cantidad de niños y niñas sin documento nacional de identidad, lo que a su vez está relacionado con la cantidad de inmigrantes que habitan ambas delegaciones, especialmente personas de Bolivia y Paraguay. Esta doble condición de ser inmigrante e indocumentado está naturalizada incluso para las instituciones más tradicionales como las escuelas, que no cierran sus puertas a los chicos o chicas no identificados. Sin embargo, el DNI se transforma en una traba para el acceso a pensiones 0 asignaciones sociales. 


\section{Redes institucionales, charlando sobre la corresponsabilidad}

Desde la investigación apareció como primordial analizar las representaciones que las instituciones tenían sobre el marco normativo de la Ley 13298 y cómo sus ejes configuraban el accionar de las mismas. La corresponsabilidad de la sociedad civil es uno de los puntos más importantes del paradigma.

Tanto en Villa Elvira como en Altos de San Lorenzo, al ser delegaciones con problemáticas sociales y estructurales, existe alta presencia institucional, sobretodo de instituciones como la Universidad Nacional de La Plata, así como recursos provinciales y nacionales que se despliegan allí. Observamos más concentración y diálogo institucional en Altos de San Lorenzo, lo que es atribuido en parte al tamaño del territorio. Mientras que en Villa Elvira notamos pequeñas concentraciones distribuidas a lo largo de la amplia delegación. En ambos casos funcionan las mesas barriales de niñez lo que ha sido un eslabón fundamental para pensar estos mapas de problemáticas y relaciones.

Respecto a la legitimidad institucional, encontramos que los establecimientos educativos son conocidos y reconocidos por la mayoría de la población, y los profesionales entrevistados coinciden en que las escuelas siguen teniendo gran nivel de incidencia y legitimidad social, aunque reconocen "a veces cuesta generar participación de parte de las familias" (referente de la Escuela Nro. 40).La permanencia institucional también fue una variable de análisis, se observó la intermitencia constante de personal en la mayoría de las instituciones analizadas, lo que puede explicarse, en parte, por los bajos salarios y las condiciones de trabajo. Esta situación se observa incluso en el sistema educativo, ya que las maestras que trabajan allí son las que recién comienzan pero luego eligen titularizar en otros lugares más cercanos al casco urbano.

"Como en el caso de los centros de salud, la permanencia institucional tiene que ver con la voluntad de los que aquí trabajan y no con la política institucional que es precaria y no cumple con las expectativas de la población" (Psicóloga del CPA).

Se ha observado una clara tensión entre la voluntad de las trabajadoras y los marcos burocráticos de la institución.

"Existe voluntad profesional de acomodar las prácticas a la ley pero la institución no se flexibiliza, los directivos a veces son obstáculos y es difícil aunar miradas de distintos profesionales cuando encima no 
existen recursos ni una conducción clara" (Trabajadora social del Centro de Salud Nro. 8 de Altos de San Lorenzo).

En Villa Elvira, por el contrario las trabajadoras de la Escuela 23 hace años que están en la institución y hablan de conocer las trayectorias escolares de los estudiantes en relación a las lógicas del barrio: "Somos un referente en el barrio, ante problemáticas las familias recurren aquí".

Existen dos características muy mencionadas por las entrevistadas de la escuela y es la importancia de directivos con objetivos territoriales claros y comprometidos sumado a la capacidad de articulación para la resolución de casos. Cuestiones que al estar fortalecidas en la institución antes mencionada, hacen que, a pesar de que la misma no tenga una comunicación directa con la comunidad, los padres participen y se sientan interpelados.

Dentro de las instituciones más referenciadas en las delegaciones, podemos resaltar en Altos de San Lorenzo, el protagonismo del programa provincial Barrio Adentro (10). El ingreso de los talleristas y profesionales al barrio fue a través de referentes de comedores, realizan seguimiento en la resolución de situaciones y se evidencia contacto directo y fluido con las familias. Como estrategia de abordaje, un concepto clave para pensar la promoción, protección y restitución de derechos, los trabajadores de Barrio Adentro hablan de generar "dispositivos flexibles", es decir pensar en actividades en el marco de las lógicas territoriales y no imponer formas de hacer. "Se busca la integración social en base a la comprensión socio-histórica de casos particulares" (Psicóloga del programa Barrio Adentro).

En este sentido, se observan como referentes instituciones que valoran los espacios que son elegidos por los chicos. Cabe destacar, que si bien son organizaciones nuevas y con escasos recursos, han logrado, en algunos casos más participación y llegada a las familias que instituciones hegemónicas como la escuela o los centros de salud. Esto puede explicarse debido a que estas instituciones están más abiertas a la escucha y posible resolución de todo tipo de problemáticas del barrio. Otro de los términos que aparece en el campo, es la "metodología de abordaje" de los niños y las niñas, esto se relaciona con los ejes claves de la Ley 13298 , se observa nuevamente que las organizaciones más nuevas trabajan en pos de los lineamientos de la normativa, con cierta flexibilidad en sus acciones, momentos de escucha de los niños y las niñas y prácticas que impliquen su participación ciudadana, como pintar un mural, buscar algún tipo de solución a problemas del barrio, etc. Sin embargo, las instituciones llamadas en este trabajo "hegemónicas", como la escuela y los centros de salud siguen siendo burocráticos y poco flexibles, ante las nuevas prácticas que conlleva el nuevo paradigma. Un ejemplo lo constituyen las trabas de los directivos hacia el egreso de los equipos de orientación 
educacional al barrio o a las mesas barriales de niñez o, incluso, la no cobertura del seguro ante situaciones que sucedan fuera del establecimiento.

Varias de las trabajadoras sociales entrevistadas comentan que ellas sí trabajan desde el paradigma de promoción de derechos, sin embargo indican que existe una fisura con respecto a las prácticas de los y las maestras que no están capacitados en relación a la normativa. En la escuela 23 las trabajadoras sociales utilizan el concepto "trayectoria escolar", les piden a los docentes que vean el caso desde el recorrido biográfico del niño o la niña, esto quiere decir: saber dónde vive, qué estructura familiar tiene, si presenta problemas de salud, cuál es la situación económica, etcétera.

Uno de los puntos principales que hace a la metodología de abordaje es cómo hacer frente a la urgencia o en palabras de las referentes "cómo se tramita la urgencia". Las instituciones como Barrio Adentro sostienen que ante la urgencia hay que buscar la articulación entre los referentes del barrio y las organizaciones y resaltan la necesidad de generar espacios de intervención y participación para evitar las urgencias. Existen organismos que nacen de la emergencia. Un ejemplo es la Unión de Cartoneros, cuya referente señala: "no hay que sentir impotencia ante el no poder hacer en el inmediato, ser paciente, saber escuchar y saber articular para intentar resolver".

Existe un extenso debate en torno a si se trabaja en la prevención, la promoción o la restitución de derechos. Las primeras dos etapas hacen referencia a la anticipación y la divulgación, mientras que la última implica actuar cuando el derecho ya fue vulnerado y hay que restituirlo. La mayoría de las instituciones relevadas trabaja desde la promoción, excepto el Centro de Prevención de Adicciones que atiende situaciones de consumo por iniciativa de la familia o de los mismos jóvenes. Las situaciones que llegan a las mesas barriales de niñez son situaciones que ya tienen derechos vulnerados, las mesas trabajan desde la articulación pero para lograr la restitución. "La escuela siempre termina manteniendo la red, somos los que estamos constantemente con los niños" (Trabajadora Social de la Escuela 125). 


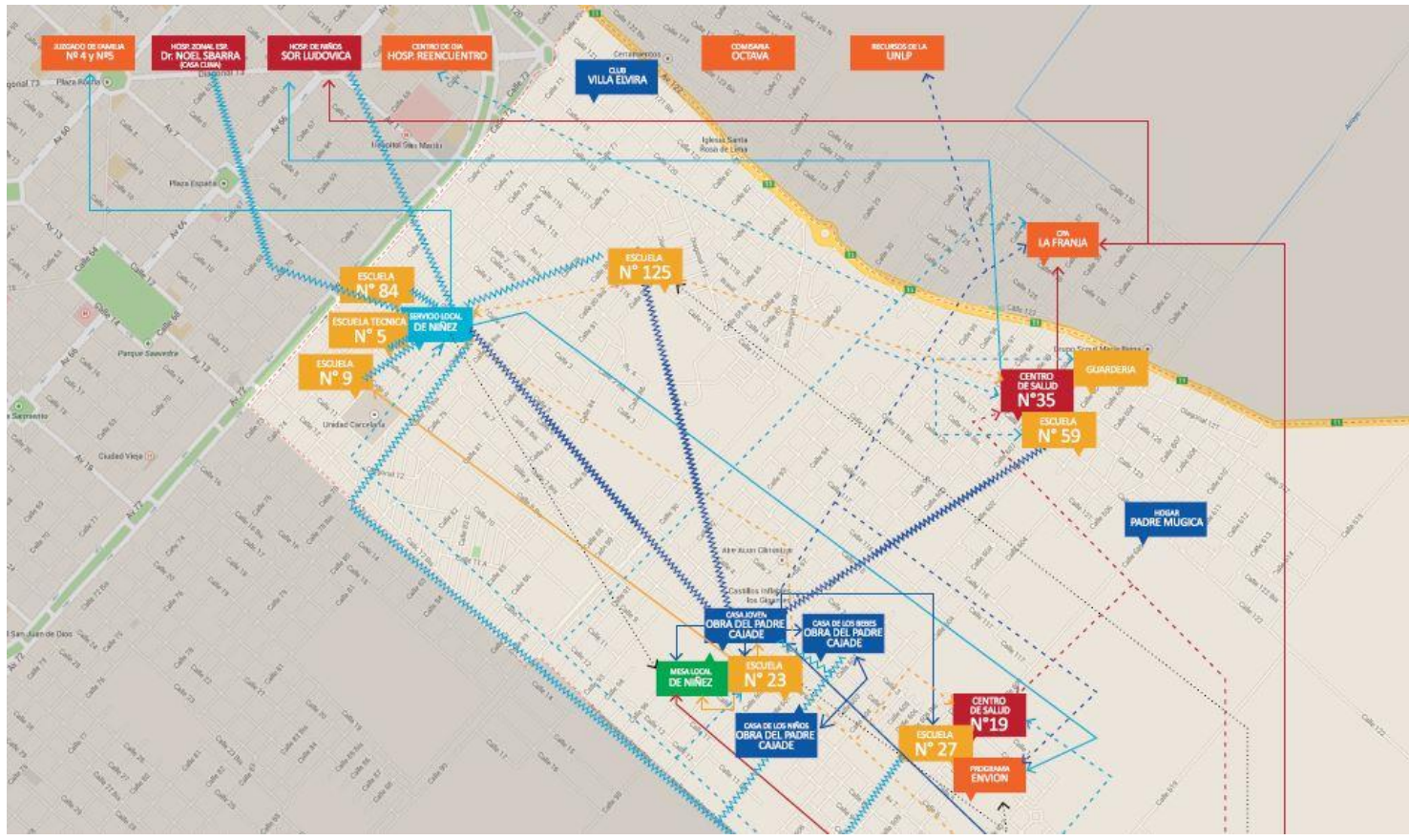

Figura 7. Mapeo de producción propia de las relaciones institucionales en Villa Elvira.

\section{Representación de las prácticas de los Servicios locales de Niñez de Altos de San Lorenzo y Villa Elvira}

El objetivo final del trabajo de investigación es poder describir y analizar las prácticas que despliegan los Servicios locales y sobre qué representaciones de la niñez, del barrio y de su trabajo lo hacen. Si bien la amplitud de los hallazgos no puede esbozarse en este trabajo, las aristas centrales que definieron los diferentes abordajes son las siguientes:

- Metodología de abordaje configurada por la noción de derecho vulnerado.

- Derecho vulnerado como aporte de "legalidad" al tratamiento de la situación.

- La urgencia determina el "recorte de la demanda".

- Diferenciación de las situaciones de derecho vulnerado "legales de las territoriales".

- Instancia de restitución de derechos. Lo que determina la mirada en torno a la niñez.

Para entender las prácticas, es necesario pensar cómo los Servicios Locales comprenden su labor en relación con la configuración que realizan de la niñez. 
"Los vemos como sujetos de derechos, cuando abordamos un caso, vemos si el chico está atravesado por las instituciones, en especial lo que respecta a la salud y educación" (psicóloga del SLPPD de Altos de San Lorenzo).

En este sentido, se puede afirmar que los Servicios Locales no realizan actividades desde la promoción o la prevención, sino que trabajan la restitución. Por lo que su mirada respecto a la niñez es sesgada, se limita a los niños y niñas que ya tienen derechos vulnerados, hablamos de la infancia que debe ser protegida o los niños o niñas mal llamados "problemáticos". Este dato que puede parecer una obviedad, va configurando las prácticas de los actores de la Dirección de Niñez y cala hondo en la planificación de políticas públicas. ¿Qué implica diseñar políticas públicas pensadas para sujetos a los que hay que proteger? La reflexión nos lleva a pensar a los niños y las niñas como objetos de protección tal como sucedía en el Sistema de Patronato. En este sentido, es necesario aclarar que la Ley establece que los Servicios Locales tendrán que tener un área de atención de casos y un área de promoción. En el municipio de La Plata nunca se creó el Área de Promoción de Derechos, sino que se ejecutan algunos programas provinciales como el ENVION, que no sólo han sufrido recortes sino que no están pensando en los jóvenes como sujetos de derechosm(11). Detrás de esta configuración sobre la niñez que sedimenta en los territorios, se esconde esta pregunta: “¿Qué contradicciones surgen de la aplicación de una legislación que presupone la igualdad en abstracto de todos los niños, niñas y adolescentes, en términos del interés superior del niño, en una sociedad profundamente desigual?" (Ferioli y Violini, 2011: 5).

Esta representación sobre la niñez se refuerza aún más con la noción tan discutida sobre el "derecho vulnerado". A partir de la existencia o no de derecho vulnerado es que los Servicios Locales "toman el caso", es decir crean un legajo y abordan la situación particular.

"Antes, cuando los casos llegaban acá, todos [los casos] se tomaban, no se visualizaba en cual había derecho vulnerado, esa fue una cuestión que surgió acá" (Psicóloga del SLPPD de Villa Elvira).

"Si no tienen legajo, no los tratan a los casos y para hacer el legajo nos piden informes complejos, que no sabemos bien que es lo que buscan con ellos, es una de dar vueltas con papeles terrible" (Trabajadora Social Escuela Nro. 40).

La pregunta sobre cuándo existe derecho vulnerado, es una que atraviesa todas las instituciones y que los Servicios intentan terminar de delimitar. Por ejemplo, para la escuela el ausentismo es derecho vulnerado, mientras que para los Servicios locales está relacionado con la "denuncia", es decir con alguna situación extrema como abuso o maltrato familiar o situación de calle. El recorte sobre el derecho vulnerado sumado a la urgencia con la que se presentan 
las situaciones, implica volver a la judicialización de las prácticas, ya que se "recorta la demanda", privilegiando las situaciones con bagaje "legal" -por ejemplo un pedido de juzgado o una citación de Servicio- de las situaciones con urgencia "territorial", por ejemplo un pedido de una escuela o de alguna familia. Esta situación es la que complejiza la corresponsabilidad en el abordaje entre las instituciones y los Servicios locales.

Esta representación configura la metodología de abordaje, que en muchas oportunidades se reduce a las acciones o demandas de los juzgados- que tienen plazos asignados- y a su acatamiento por parte de los Servicios locales. Se agrava aún más cuando los casos son mediáticos, como ha sucedido en ambas delegaciones, en las que deben centrarse en esa situación ante el impacto político que podría tener.

"La verdad es que nosotros actuamos como auxiliares de la justicia. En realidad cuando nos llaman agachamos la cabeza y actuamos, que se yo, tal vez esto no está mal, el juez tiene la facultad" (Psicóloga del SLPPD de Altos de San Lorenzo).

Mientras que para las organizaciones e instituciones mencionadas las estrategia es el trabajo en red, para los Servicios Locales se trata de la planificación de su accionar. En el caso del Servicio local de Villa Elvira, tienen delineado cuáles son los momentos a seguir y ninguno contempla la articulación con organizaciones.

\begin{tabular}{|c|c|c|}
\hline $\begin{array}{l}\text { Citación a una entrevista } \\
\text { con la psicóloga del SLPPD. } \\
\text { (Operador) } \\
\end{array}$ & $\rightarrow 2 \begin{array}{l}\text { Entrevista en el local del } \\
\text { SLPPD con la psicóloga. } \\
\text { (Psicóloga) }\end{array}$ & $\begin{array}{l}\text { Visita al barrio para ver la } \\
\text { situación que atraviesa la } \\
\text { familia, el territorio en el } \\
\text { que vive y los recursos }\end{array}$ \\
\hline & $\begin{array}{l}\text { En el caso de que sea } \\
\text { necesaria una medida } \\
\text { judicial o el caso venga de } \\
\text { una denuncia de la justicia, } \\
\text { interviene el Abogado. }\end{array}$ & $\begin{array}{l}\text { Seguimiento y acompaña- } \\
\text { miento en el tiempo. } \\
\text { (Equipo) }\end{array}$ \\
\hline
\end{tabular}

Figura 8. Momentos de trabajo del Servicio Local de Villa Elvira (producción propia).

Ya cerca de las conclusiones, es necesario aclarar que estos Servicios están obligados al "recorte de la demanda" debido a la escasez de recursos que no sólo se observa en los recursos humanos sino también en las condiciones estructurales de los Servicios y de las condiciones habitacionales de los niños y niñas que abordan. En este sentido, no existen convenios con otras áreas municipales que puedan aportarles recursos materiales para trabajar en la restitución de los derechos de forma interdisciplinaria. Por el contrario, los Servicios 
acuden a recursos provinciales. El no tratamiento de situaciones territoriales, los lleva, sobre todo en el caso de Villa Elvira, a tener una relación conflictiva con las organizaciones territoriales.

\section{Algunas conclusiones y posibles sugerencias}

Del trabajo de campo surge que los términos que establece la normativa están reconocidos y son empleados por todos los actores sociales entrevistados y observados. Existe un empleo del lenguaje que está pensando al niño o la niña como sujeto de derechos, su interés superior, la importancia del territorio e instituciones como la escuela o los centros de salud que hacen a su identidad. Sin embargo, en las prácticas existe una diversidad en la apropiación de estos términos que configura distintos mecanismos de acción. Esto puede explicarse, ya que son prácticas instituyentes, nuevos organismos que no sólo deben desandar ochenta años del Sistema de Patronato sino que lo deben hacer con mínimos recursos.

Una de las conclusiones que relacionamos con la descentralización estatal y con el corrimiento de lo judicial a lo administrativo, ergo de los juzgados al territorio, es que si bien no existen acuerdos entre las organizaciones del territorio y las unidades operativas técnicas, es decir los Servicios locales, las primeras le reclaman su liderazgo. Existe por parte de los actores un reconocimiento hacia la institución como referente, pero hay una dificultad por parte de los Servicios de hacerse cargo de ese rol. Si bien muchas de las organizaciones nacieron por el vacío estatal, reconocen el nuevo rol del estado a partir de la descentralización y lo buscan, lo demandan, le exigen.

Otra de las conclusiones o puntos de inicio para pensar políticas públicas es que si bien el recurso es un limitante concreto en todas las aristas de las prácticas de los Servicios locales, desde imprimir una citación hasta trasladar a un niño o niña a un hogar convivencial, las organizaciones reclaman sobre todo por el método. Exigen establecer acuerdos en la metodología, poder articular la "estrategia de abordaje", tema que se debate en las mesas locales de niñez. La inclusión en la mesa técnica de Altos de San Lorenzo y la mesa barrial de Villa Elvira fueron fundamentales para pensar en profundidad cuestiones técnicas respecto a la temática como así también problemáticas barriales que repercuten en los derechos de la niñez. Se observa que ambos Servicios Locales no tienen como política la comunicación con la franja etaria a la que abordan, es decir los niños y niñas. No existen debates sobre cómo acercarse a ellos y no constituyen esas nociones parte de la metodología de abordaje. Uno de los grandes cambios del paradigma es entender a los niños como sujetos de derechos, para ello nada más 
preponderante que garantizar la escucha y no sólo desde una entrevista psicológica individualizada, sino primando las posibilidades de interactuar desde sus cotidianidades, conociendo sus recorridos, sus espacios y trayectorias comunes.

Por último, algunos de los hallazgos de la investigación han sido el rol de la mujer en la construcción de la niñez y en la representación de estas nuevas prácticas, la preponderancia de las mesas como lugares de articulación y el barrio como estructurante fundamental de las prácticas.

Entre las propuestas respecto a políticas públicas se sugiere el fortalecimiento de las mesas barriales de niñez para la producción conjunta de guías para producir informes que se eleven a los Servicios Locales, que planteen estrategias conjuntas. Así como el fortalecimiento de los Consejos locales de Niñez municipales (12). Es sumamente necesario que el municipio genere estadísticas para proyectar políticas públicas con presupuesto municipal y evitar la dependencia casi absoluta de los recursos provinciales. Por último, que los SLPPD se transformen en líderes mediante la realización de capacitaciones y talleres con organizaciones del barrio. Que lideren procesos de construcción territoriales y diseñen de jornadas con niños y niñas donde los SLPPD puedan conocer a los sujetos que abordan desde lugares recreativos y lúdicos que ponderen su voz desde la promoción.

\section{Notas}

(1) Diferentes representaciones de la pobreza infantil en Argentina (2010-2017). https://bit.ly/2Ra5onP

(2) Decisión Administrativa 1605/2018: https://bit.ly/2SDJWUE

(3) Alerta y preocupación por el recorte de presupuesto en el SENNAF, en https://bit.ly/2QoP2m2

(4) Las estadísticas fueron analizadas y sistematizadas por Norberto Liwski en el Encuentro de Consejos Locales de Niñez de Bahía Blanca 2018. Disponible en https://bit.ly/2GTVQZl

(5) Es el último censo que presenta la división habitacional por delegaciones.

(6) Durante 2016, el Servicio local de Villa Elvira se mudó a la Delegación en 7 y 82, mientras que el Servicio local de Altos dejó su sede en territorio y se mudó a la Dirección, en el centro de La Plata. A fines de 2017 y después de muchas presentaciones de las organizaciones, se encuentra nuevamente en territorio. Direcciones de todos los Servicios disponibles aquí: https://goo.gl/HrJj9V

(7) Durante la investigación se hicieron un par de salidas con el operador de calle a repartir citaciones y poder chalar con las familias. Según las trabajadoras del Servicio local fue despedido por la cantidad de faltas y porque "no hacía lo que tenía que hacer". Durante la investigación observamos que se trataba del más comprometido en relación a la temática y a las situaciones en el barrio. Era el único que participaba en la mesa local de niñez. Desde ese órgano barrial presentaron varias notas a la Dirección de Niñez pidiendo explicación ante su abrupto despido.

(8) Libro y anexos, en https://goo.gl/HQiRfc y https://goo.gl/Rv8npD

(9) Mapas de relaciones institucionales, en https://goo.gl/yWa4wQ 
(10) En 2016 el Programa provincial Barrio Adentro se mudó, por directivas del Organismo Provincial, a la delegación de San Carlos, lo que impactó en la articulación organizacional.

(11) Lo evidencia la no permanencia de los adolescentes en dichos espacios.

(12) El Consejo Local de Niñez es un organismo creado por la Ley 13298 que tiene como objetivo la participación de las organizaciones en el diseño y monitoreo de políticas públicas.

\section{Bibliografía}

Aréchaga, A. J. (2011). El cuerpo y el espacio social. Revista Question, 1(31), Universidad Nacional de La Plata, Argentina. Recuperado de https://perio.unlp.edu.ar/ojs/index.php/question/article/view/1210

Bourdieu, P. (1991). El sentido práctico. Madrid: Taurus.

Carballeda, A. (2008). Los cuerpos fragmentados. La intervención en lo social en los escenarios de la exclusión y el desencanto. Buenos Aires: Paidos.

Castells, M. (1999). Globalización, Identidad y Estado en América Latina. Ponencia de Programa de las Naciones Unidas para el Desarrollo, Chile.

Ferioli, A. y Violini, M. B. (2011). Repensando las categorías hegemónicas de niñez, adolescencia y juventud. Ficha de Cátedra de la Facultad de Trabajo Social de la UBA. Recuperado de http://www.trabajosocial.fsoc.uba.ar/jornadas/contenidos/37.pdf

García Canclini, N. (1996). Culturas Hibridas y estrategias comunicacionales. Ponencia en el Seminario Fronteras culturales: Identidad y Comunicación en América Latina, Universidad de Stirling.

González, J. (1992). Introducción: las perspectivas panorámicas y políticas cartográficas. Recuperado de https://bit.ly/2dvNDK7

Grimson, A. (2003). Las culturas son más híbridas que las identificaciones. Diálogos interantropológicos. Recuperado de https://bit.ly/2AFwwRp

Ley de la Promoción y Protección Integral de los Derechos de los niños y adolescentes de la provincia de Buenos Aires, Nro. 13298. Legislatura de la provincia de Buenos Aires, Argentina, 2007.

Ley Nacional de Protección Integral de los derechos de las niñas, niños y adolescentes, Nro. 26061. Congreso de la Nación, Argentina, 2005.

Martin-Barbero, J. (1987). De los medios a las mediaciones. Comunicación, cultura y hegemonía. Santa Fe de Bogotá: Convenio Andrés Bello.

Massoni, S. (2017). Medir la comunicación desde lo vivo. Indicadores comunicacionales en dimensiones múltiples. Rosario: UNR Editora.

Question, Vol. 1, N. 61, enero-marzo 2019. ISSN 1669-6581

Instituto de Investigaciones en Comunicación | Facultad de Periodismo y Comunicación Social | Universidad Nacional de La Plata La 
Prieto Castillo, D. (1990). Diagnóstico de comunicación. Quito: CIESPAL.

Schmucler, H. (1984). Un proyecto de Comunicación/Cultura. Revista Comunicación y Cultura, 12, México.

Uranga, W. (2012). Intervenir: reflexiones desde la comunicación. Cuadernos de cátedra Nro. 1. Taller de Planificación de Procesos Comunicacionales, Facultad de Periodismo y Comunicación Social, UNLP.

Uranga, W; Bruno, D. y Vargas, T. (2012). Diseño estratégico. Cuadernos de cátedra Nro. 7. Taller de Planificación de Procesos Comunicacionales, Facultad de Periodismo y Comunicación Social, UNLP.

Uranga, W. (2007). Mirar desde la comunicación. Una manera de analizar las prácticas sociales. Taller de Planificación de Procesos Comunicacionales, Facultad de Periodismo y Comunicación Social, UNLP. 